\title{
Resistenza a fatica di strutture in leghe di alluminio: normative a confronto e verifica sperimentale
}

\author{
B. Atzori, G. Meneghetti, B. Rossi \\ Università di Padova, Dipartimento di Ingegneria Meccanica,bruno.atzori@unipd.it
}

\begin{abstract}
RIASSUNTO. Le problematiche relative all'utilizzo delle normative nell'ambito della progettazione a fatica di strutture in leghe di alluminio sono state di recente affrontate in numerosi lavori che hanno messo in luce sia le difficoltà legate al passaggio da normative oramai obsolete ad altre di nuova concezione, ma di caratteristiche e struttura completamente diverse, che la sostanziale carenza anche all'interno delle più recenti normative europee, quale l'Eurocodice 9, di molti risultati e metodi sviluppati in anni di ricerca scientifica e ormai indiscutibilmente consolidati. Il presente lavoro si propone di approfondire entrambe le tematiche tramite il confronto tra risultati sperimentali tratti da letteratura e le corrispondenti curve di resistenza proposte rispettivamente dalla normativa italiana UNI 8634, di recente ritirata, e dall'Eurocodice 9. In questo modo verranno messe in luce le differenze tra i valori di resistenza proposti dalle due norme e verrà illustrata sia la corrispondenza a volte poco soddisfacente con i risultati sperimentali che le conseguenze dovute alla mancata applicazione di assodati risultati teorici.
\end{abstract}

\begin{abstract}
The employment of standard codes in fatigue design of aluminium alloy structures has been the subject of many recent papers where both the comparison among different design standards and the lack of application, even in the latest European codes, of reliable theoretical results were discussed. The aim of this work is to deepen the comparison between the Italian code UNI 8634, recently withdrawn, and the European standard Eurocode 9, published in 2007, through the comparison of experimental data taken from literature with the corresponding design curves proposed by both of codes. In this way, the differences between the fatigue resistance data will be pointed out together with the effects of neglecting well known and reliable theoretical results.
\end{abstract}

Parole Chiave. Lega leggera; Fatica; Giunti saldati; Normative; Dati sperimentali

\section{INTRODUZIONE}

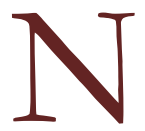

ell'ambito della progettazione di strutture in leghe di alluminio, una delle prime normative in assoluto a presentare dati relativi alla resistenza a fatica di particolari strutturali di diverso tipo è stata la normativa italiana UNI 8634 [1]. Tale norma, emanata nel 1985 e rimasta in vigore fino a dicembre del 2008, si basava sulla vasta attività di ricerca e rianalisi di dati sperimentali effettuata tra il 1975 ed il 1980 dal Prof. Atzori prima presso il Laboratorium für Betriebsfestigkeit di Darmstadt e poi presso l'università di Bari [2-4]. Tali ricerche avevano condotto, su analogia di quanto già ottenuto dal prof. Haibach per le giunzioni in acciaio, alla definizione di una banda di dispersione unificata (Fig. 1) capace di interpretare i risultati di prove di fatica su giunti di geometrie e dimensioni assolute diverse, purché la cricca si inneschi al piede del cordone di saldatura. Tale risultato [3] era stato assunto come principio base della normativa italiana, tenendo conto che la diversa tipologia di giunto causa una semplice traslazione verticale della banda di dispersione se le tensioni considerate sono quelle nominali calcolate sulla lamiera principale.

La UNI 8634, che per facilitare i progettisti riproduceva la normativa per le strutture in acciaio allora in vigore [5] non venne mai aggiornata per mantenere la corrispondenza con la successiva versione della UNI 10011 sugli acciai [6] in 
quanto alla fine degli anni '80 venne istituita una commissione CEN per la stesura di una normativa europea su tematiche analoghe. I lavori portarono nel 2002 all'emissione di una prenorma [7] ed infine nel 2007 all'emissione della norma definitiva [8], che dopo il ritiro della UNI 8634 si configura a livello italiano, oltre che europeo, come normativa di riferimento per la progettazione a fatica di strutture in lega leggera. Al fine di mantenere una certa omogeneità nella presentazione dei risultati, l'Eurocodice 9 riproduce la struttura adottata dall'Eurocodice 3 per gli acciai [9] e risulta pertanto avere un formato notevolmente diverso rispetto alla normativa italiana.

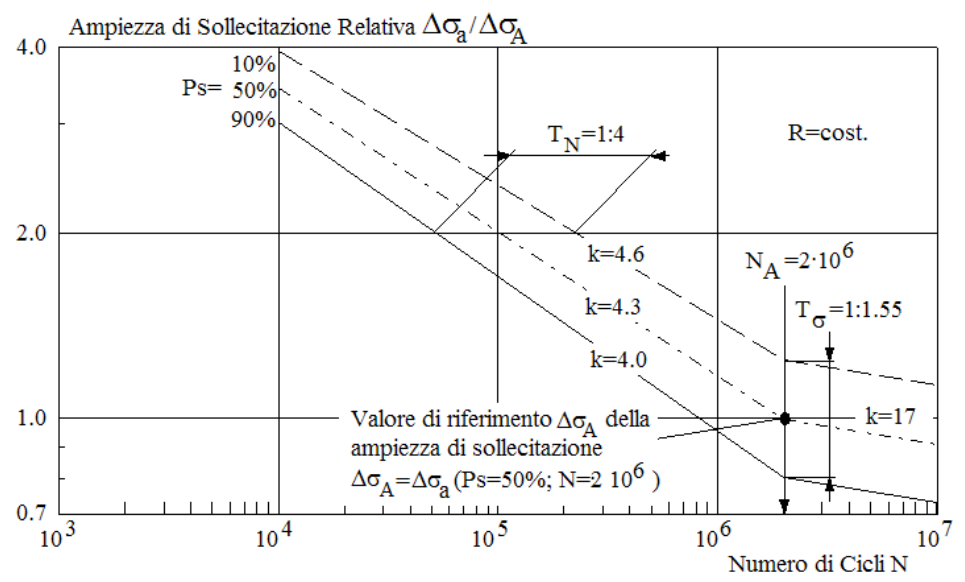

Figura 1: Banda di dispersione relativa alle giunzioni saldate in lega leggera [2].

Le difficoltà presenti nel passaggio da una normativa all'altra sono state messe in luce in recenti lavori [10, 11] sia per quanto riguarda la corrispondenza tra i dettagli strutturali che dal punto di vista dei valori di resistenza. Inoltre, durante il lungo periodo di stesura dell'Eurocodice 9, le analisi teoriche nell'ambito delle giunzioni saldate hanno avuto notevolissimo impulso, soprattutto dopo la scelta di considerare il piede del cordone di saldatura, nel caso di cordoni d'angolo, come un intaglio acuto con raggio di raccordo nullo e di assumere per il cordone uno spessore ed un'inclinazione di $45^{\circ}$ costanti $[12,13]$ che ha portato a legare le problematiche delle giunzioni saldate a quelle degli intagli acuti in generale. Numerose analisi sono state sviluppate su queste basi [14-20] negli anni successivi portando, tra i molteplici risultati, a definire, per giunti con cordoni d'angolo e rottura a piede cordone, un'unica banda di dispersione unificata (Fig. 2, [20]), con valori di pendenza e dispersione molto prossimi a quelli determinati in passato (Fig. 1) ma che esprimendo in punti sperimentali in funzione non delle tensioni nominali, bensì delle tensioni locali immediatamente prossime alla zona di innesco cricca tramite l'utilizzo del parametro locale $\Delta \mathrm{K}_{1} \mathrm{~N}$, ovvero fattore di intensificazione delle tensioni di intaglio di modo I, elimina la traslazione verticale dovuta alle differenti concentrazioni di tensione strutturale. Un'espressione conveniente del fattore di intensificazione delle tensioni di intaglio di modo I per i giunti saldati [14] risulta essere:

$$
\Delta \mathrm{K}_{1} \mathrm{~N}=\mathrm{k}_{1} \Delta \sigma_{\mathrm{n}} \mathrm{t}^{0.326}
$$

dove $\mathrm{k}_{1}$ è un coefficiente adimensionale, analogo al coefficiente teorico di concentrazione delle tensioni $\mathrm{k}_{\mathrm{t}}$, che dipende dalla geometria delle parti collegate e del cordone di saldatura stesso, $\Delta \sigma_{\mathrm{n}}$ è il range di tensione nominale applicata, $\mathrm{t}$ lo spessore del piatto principale caricato, 0.326 esponente valido nell'ipotesi di cordone di saldatura schematizzato come intaglio con angolo di apertura $135^{\circ}$ (caso tipico di cordone d'angolo). Si noti come l'Eq. 1 permetta una valutazione ed una formalizzazione accurata dell'effetto scala, prima difficilmente descrivibile in termini rigorosi.

In questo modo l' approccio allo studio della resistenza a fatica di giunzioni saldate basato sulle tensioni nominali è stato soppiantato dallo studio dell'effettivo campo di tensione locale che è stato interpretato anche come sovrapposizione dell'intaglio acuto al piede del cordone di saldatura e dell'intaglio strutturale dovuto alla geometria complessiva della giunzione [21].

Se i risultati teorici qui brevemente riassunti non erano presenti, per ovvie questioni temporali, nella UNI 8634, risultano molto poco recepiti anche dall'Eurocodice 9, che come evidenziato da recenti lavori [22, 23] sottostima notevolmente 
l'effetto scala considerandolo solo in un numero molto limitato di casi e soprattutto ignora completamente l'utilizzo di approcci di tipo locale, prevedendo al più l'uso di metodi di tipo "hot spot" che analizzano però il solo campo di tensione strutturale dovuto alla geometria complessiva della giunzione escludendo invece gli effetti di intaglio acuto al piede del cordone di saldatura.

Nella memoria verrà ripresa nei tratti salienti la descrizione di entrambe le normative (UNI 8634 e Eurocodice 9) mentre verrà dato ampio spazio al confronto delle curve di progettazione con i risultati sperimentali, evidenziando in particolare le incongruenze e le conseguenze delle lacune rispetto alle analisi teoriche.

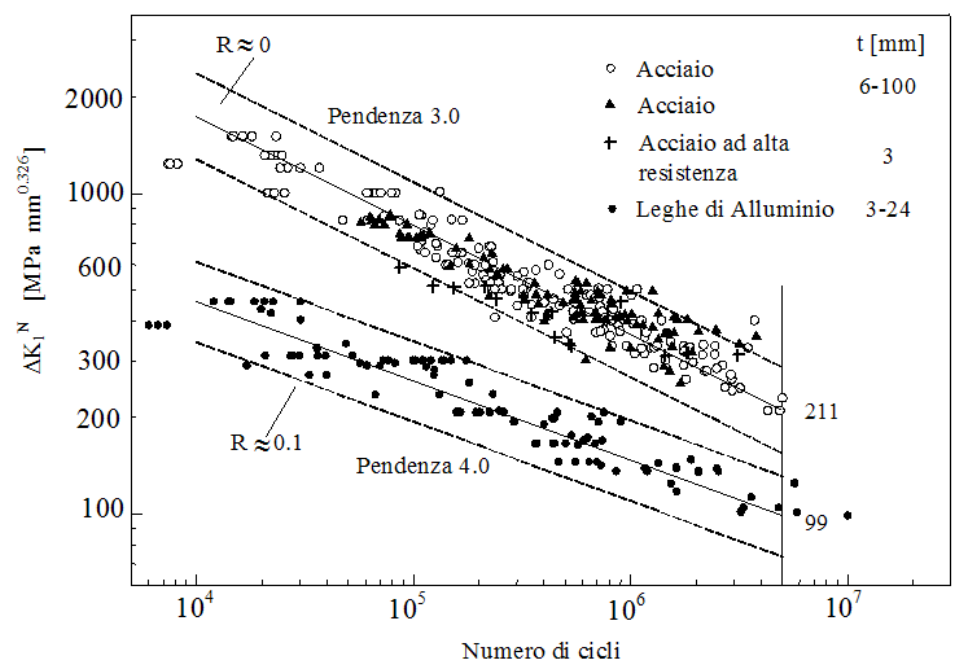

Figura 2: Resistenza a fatica di giunti a croce in acciaio e lega leggera in funzione del fattore di intensificazione delle tensioni di intaglio di modo I $\Delta \mathrm{K}_{1}{ }^{\mathrm{N}}[20]$.

\section{NORMATIVE DI PROGETTAZIONE}

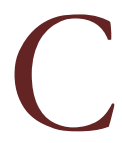

ome anticipato al paragrafo precedente, la normativa italiana UNI 8634, sintetizza i dati di resistenza a fatica relativi a prove effettuate su provini di geometria diversa sulla base del concetto di banda di dispersione unificata, sviluppato negli anni precedenti. Viene così presentata un'unica curva di resistenza a fatica (Fig. 3) che riporta in scale relative, ovvero riferite alle coordinate del ginocchio della curva stessa, l'ampiezza di sollecitazione $\mathrm{f}_{\mathrm{d}, \mathrm{a}}=\left(\sigma_{\max }\right.$ $\left.\sigma_{\min }\right) / 2$ al variare del numero di cicli n. In questa forma generale la curva è valida per tutti i materiali, i tipi di giunto e le condizioni di sollecitazione e va particolarizzata in base al tipo di lega, alla geometria del collegamento e al rapporto di sollecitazione dello specifico caso considerato tramite gli opportuni valori numerici. In base alla tipologia di giunto considerata (la normativa identifica 7 gruppi corrispondenti ad altrettante geometrie) si ottiene così una famiglia di curve aventi tutte la medesima pendenza $\left(k^{\prime}=4.3\right)$ ma che traslano nel piano $\sigma$-n per tener conto dei diversi effetti di concentrazione delle tensioni dovuti alle diverse geometrie strutturali. In particolare la normativa fornisce (Tab. 1) per ciascuno dei gruppi considerati, la posizione del ginocchio $\mathrm{n}_{\mathrm{G}}$, il corrispondente valore della resistenza di progetto $\mathrm{f}_{\mathrm{d},-1(\mathrm{n}=}$

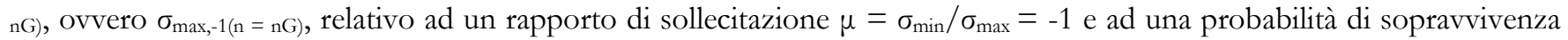
del 97.7\% (media meno due deviazioni standard) nonché il valore della resistenza statica $\mathrm{f}_{\mathrm{t}}$ Noti tali dati è immediato risalire, tramite un tradizionale diagramma di Smith, al valore della resistenza di progetto $f_{d, \mu}(n=n G)$ relativa al particolare rapporto di sollecitazione considerato e quindi al valore dell'ampiezza $\mathrm{f}_{\mathrm{d}, \mathrm{a}}(\mathrm{n}=\mathrm{nG})$.

Mentre, come già osservato, la pendenza della curva rimane la medesima per tutte la tipologie di dettagli strutturali, viceversa la posizione del ginocchio della curva varia. Inoltre non si considera definibile un limite di fatica. Per ovvie ragioni di data di emanazione, la UNI 8634 non tiene conto dell'effetto delle dimensioni assolute dei giunti, né prevede la possibilità di utilizzo di approcci di tipo alternativo a quello in tensioni nominali nel caso di geometrie complesse. Viceversa, essendo la normativa basata principalmente su prove eseguite su giunti saldati semplici, essa ritiene la resistenza 
a fatica dipendente dal rapporto di sollecitazione e dal tipo di lega impiegata, aspetti che in seguito sono stati invece ritenuti ininfluenti sul comportamento a fatica [24].

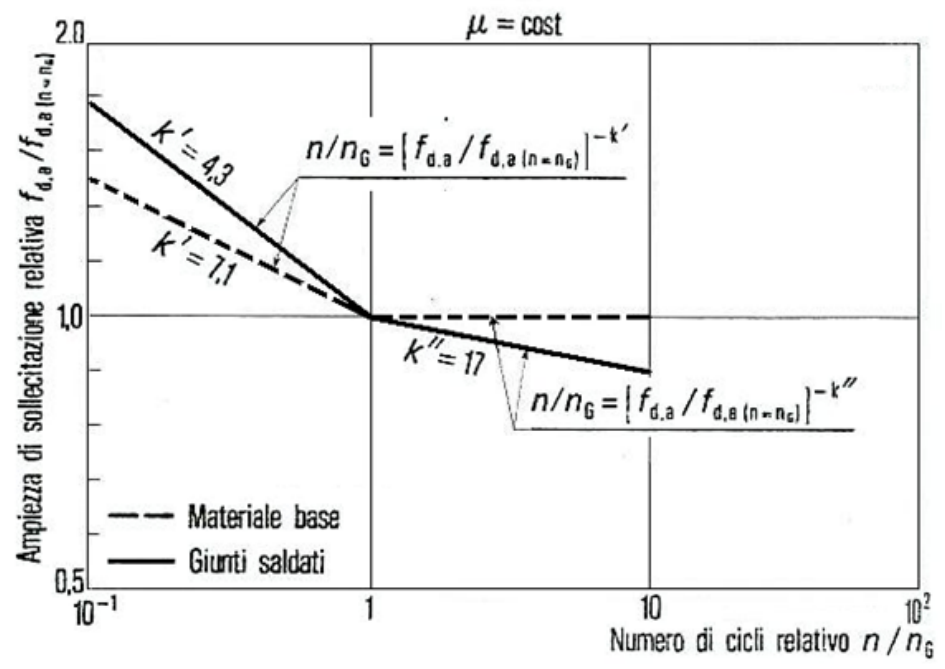

Figura 3: Curva di resistenza a fatica per giunti saldati in lega d'alluminio secondo UNI 8634 [1].

\begin{tabular}{llll}
\hline Gruppo & $\mathbf{n}_{\mathbf{G}}$ & $\mathbf{f}_{\mathrm{d},-1(\mathrm{n}=\mathrm{nG})}[\mathbf{M P a}]$ & $\mathbf{f}_{\mathbf{t}}[\mathbf{M P a}]$ \\
$\mathrm{A}$ & $2 \cdot 10^{6}$ & $83^{*}$ & $278^{*}$ \\
$\mathrm{~B}$ & $2 \cdot 10^{6}$ & 43 & $278^{*}$ \\
$\mathrm{C}$ & $2 \cdot 10^{6}$ & 38 & $278^{*}$ \\
$\mathrm{D}$ & $2 \cdot 10^{6}$ & 31 & $278^{*}$ \\
$\mathrm{E}$ & $2 \cdot 10^{6}$ & 27 & $278^{*}$ \\
$\mathrm{~F}$ & $3.2 \cdot 10^{6}$ & 20 & $278^{*}$ \\
$\mathrm{G}$ & $10^{7}$ & 10 & $278^{*}$ \\
\hline
\end{tabular}

Tabella 1: posizione del ginocchio della curva di Woehler e valori di resistenza per i diversi gruppi di giunti considerati nell’UNI 8634 (*= valore medio, per i valori precisi riferiti ai vari tipi di lega si rimanda al prospetto 53 della normativa).

Molto diverso dalla UNI 8634 già per quanto riguarda l'impostazione di base, l' Eurocodice 9 fa riferimento a dati di resistenza a fatica relativi a risultati ottenuti su strutture reali, e non su provini come nel caso della norma italiana, sintetizzandoli in curve standard, la cui forma generale è illustrata in Fig. 4. Tale curva descrive, su scala doppio logaritmica e con riferimento ad una probabilità di sopravvivenza del $97.7 \%$, l'andamento della resistenza a fatica in termini di range di tensione $\left(\Delta \sigma=\sigma_{\max }-\sigma_{\min }\right)$, in funzione del numero di cicli $\mathrm{N}$.

$\mathrm{Nel}$ diagramma, si possono distinguere tre punti particolari: il punto $C\left(\mathrm{~N}_{C}=2 \cdot 10^{6} \mathrm{cicli} / \Delta \sigma_{\mathrm{C}}\right)$, utilizzato come valore di riferimento per definire la categoria dei dettagli strutturali, il punto $\mathrm{D}\left(\mathrm{N}_{\mathrm{D}}=5 \cdot 10^{6} \mathrm{cicli} / \Delta \sigma_{\mathrm{D}}\right)$, limite di fatica per storie di carico ad ampiezza costante, e il punto $\mathrm{L}\left(\mathrm{N}_{\mathrm{L}}=10^{8} \mathrm{cicli} / \Delta \sigma_{\mathrm{L}}\right)$, cut-off limit, ovvero valore tale da ritenere che sollecitazioni di ampiezza inferiore ad esso non influenzino la vita a fatica del componente. La curva presenta pendenza inversa $\mathrm{m}_{1}$ nel tratto $\mathrm{N}<\mathrm{N}_{\mathrm{D}}$, pendenza $\mathrm{m}_{2}=\mathrm{m}_{1}+2$ nel tratto $\mathrm{N}_{\mathrm{D}}<\mathrm{N}<\mathrm{N}_{\mathrm{L}}$; tuttavia il valore di $\mathrm{m}_{1}$ non è standardizzato bensì varia a seconda della tipologia di giunto considerata, in disaccordo sia con la UNI che con le analisi teoriche [2, 14, 18, 23]; viceversa i valori di $\mathrm{N}_{C}, \mathrm{~N}_{\mathrm{D}}, \mathrm{N}_{\mathrm{L}}$ restano costanti per tutte le categorie di dettagli strutturali. Nell'Eurocodice la resistenza a fatica viene ritenuta indipendente dal rapporto di ciclo $\mathrm{R}=\sigma_{\min } / \sigma_{\max }$ e dal tipo di lega di alluminio (tranne nel caso di dettagli strutturali per materiale base in lega 7020). La curva di Woehler illustrata in Fig. 4 va particolareggiata con gli opportuni valori numerici a seconda della tipologia di giunto considerata. A questo scopo nell'Eurocodice viene definita una serie di valori standardizzati di $\Delta \sigma_{C}$ (ricavati da storie di carico con rapporto di ciclo $\mathrm{R} \geq 0.5$ ) riportati in Tab. 2 . In Tabella 3 vengono illustrate invece le diverse tipologie di giunti considerate dall'Eurocodice 9 e la corrispondenza con i gruppi proposti dalla normativa italiana. Si noti come la varietà di dettagli strutturali classificati sia notevolmente maggiore 
nel caso dell'Eurocodice. Inoltre mentre nella UNI 8634 ad un determinato gruppo di giunti corrisponde un solo valore di resistenza di progetto, all'interno di ciascuna tipologia di dettaglio riportata in Tab. 3 sono presenti più sotto categorie identificate da valori di resistenza $\Delta \sigma_{\mathrm{C}}$ diversi.

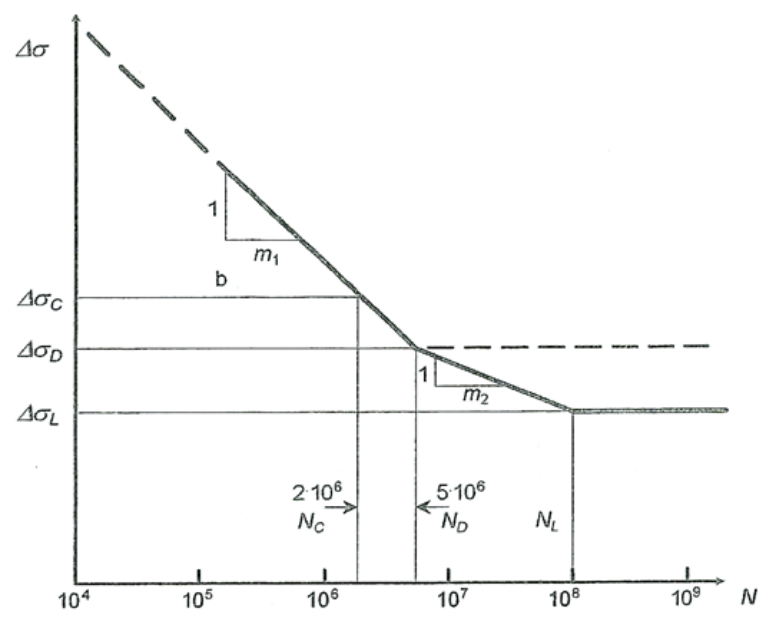

Figura 4: Curva di resistenza a fatica per giunti saldati in lega d'alluminio secondo l'Eurocodice 9 [8].

$12,14,16,18,20,23,25,28,32,36,40,45,50,56,63,71,80,90,100,112,125,140$

Tabella 2: Valori standardizzati di $\Delta \sigma_{\mathrm{C}}[\mathrm{MPa}]$ secondo l'Eurocodice 9.

\begin{tabular}{lll}
\hline Tipologia di dettagli strutturali & Eurocodice 9 & UNI 8634 \\
materiale base & Tav. J.1 & A \\
parti aggiunte tramite cordoni trasversali & Tav. J.3 & D, E, G \\
saldature longitudinali & Tav. J.5 & C \\
giunti saldati di testa & Tav. J.7 & B, D, F \\
saldature d'angolo & Tav. J.9 & F \\
collegamenti con bulloni & Tav. J.15 & - \\
giunti incollati & Tav. E.1 & - \\
travi saldate & Tav. J.11 & - \\
parti aggiunte su travi saldate & Tav. J.13 & - \\
\hline
\end{tabular}

Tabella 3: Tipologie di dettagli strutturali considerate nell' Eurocodice 9 e nella UNI 8634.

In generale nell'Eurocodice viene indicata la possibilità di rivalutare o penalizzare di una o due categorie (passando cioè al $\Delta \sigma_{\mathrm{C}}$ standardizzato immediatamente superiore o inferiore rispetto a quello caratteristico del dettaglio considerato e mantenendo invece $\mathrm{m}_{1}$ costante) alcune tipologie di dettagli strutturali. Questo modo di procedere viene applicato ad esempio per tener conto dell'effetto scala, limitatamente al caso di cordoni d'angolo non portanti, o nel caso di giunti saldati testa a testa con geometrie complesse.

Per quanto riguarda l'effetto della tensione media, l'Eurocodice ne tiene conto espressamente nel solo caso di materiale base, per il quale, nel caso di storie di carico con rapporto di ciclo $\mathrm{R}<0.5$, è possibile sostituire al valore di riferimento per la resistenza a fatica $\Delta \sigma_{\mathrm{C}}$, il valore $\Delta \sigma_{\mathrm{C}(\mathrm{R})}$ così definito:

$$
\Delta \sigma_{\mathrm{C}(\mathrm{R})}=\mathrm{f}(\mathrm{R}) \cdot \Delta \sigma_{\mathrm{C}} ;
$$

dove $f(R)$ è un fattore di incremento della resistenza a fatica che vale, limitatamente al caso di materiale base, $f(R)=1.2$ $0.4 \mathrm{R}$. Viceversa, nel caso di giunti saldati, non è previsto considerare l'effetto del rapporto di ciclo, a meno che non sia possibile determinare le tensioni residue sul componente. 
Nel caso di geometrie complesse ove l'approccio in tensioni nominali risulti difficilmente applicabile, l' Eurocodice 9 prevede l'utilizzo di un approccio di tipo hot spot da applicare nei casi di innesco cricca a piede cordone. Si tenga presente tuttavia che, come già messo in luce al paragrafo precedente, tale approccio analizza solo il campo di tensione strutturale dovuto alla geometria complessiva della giunzione, escludendo invece gli effetti dell'intaglio acuto al piede del cordone di saldatura, che risultano al contrario fondamentali per la previsione di resistenza a fatica. Viceversa non è previsto l'utilizzo di approcci di tipo locale.

\section{CONFRONTO CON DATI SPERIMENTALI}

$\mathrm{L}$ e curve di resistenza a fatica proposte dalle normative sono state confrontate con i dati sperimentali ricavati da letteratura e relativi a 15 serie di giunti di geometria diversa. Ciascuna serie di dati è stata sottoposta ad analisi statistica e sono state ricavate le curve di resistenza a fatica per una probabilità di sopravvivenza del $97.7 \%$ con confidenza 95\%. I risultati sono riportati in Fig. 5-12. In Tab. 4 si riporta invece l'intera gamma dei dati sperimentali analizzati indicando per ciascuna serie il valore di $\Delta \sigma \mathrm{N}=2 \cdot 10^{6}$, P.S. $=97.7 \%$ e la pendenza $\mathrm{k}$ della curva ricavati dall'analisi statistica. Per ciascuna serie si riportano inoltre le corrispondenti classi di resistenza fornite dalle normative e i relativi valori di $\Delta \sigma_{\mathrm{N}}=2 \cdot 10^{6}$, P.S. $=97.7 \% \mathrm{e} \mathrm{k}$. Nel caso di giunti a $\mathrm{T}$ e a croce le indicazioni riportate tra parentesi fanno riferimento alle dimensioni, in $\mathrm{mm}$, del piatto principale caricato e della lamiera trasversale, rispettivamente. Per quanto riguarda i valori di resistenza, ad esclusione del caso del materiale base, dove, come indicato al paragrafo Materiale base si è tenuto conto dell'effetto del rapporto di sollecitazione, per la UNI 8634 si è fatto riferimento ai valori relativi ad R = 0 (la maggior parte dei risultati sperimentali utilizzati per la norma italiana si riferivano infatti a $0<\mathrm{R}<0.1$ ) mentre per l'Eurocodice $\mathrm{i}$ valori di resistenza sono ricavati da dati con $\mathrm{R}>0.5$. Le serie sperimentali sono invece tutte relative a prove con $\mathrm{R} \sim 0.1$.

\section{Materiale base}

Per un corretto confronto con i dati sperimentali $(\mathrm{R}=0.1)$, i valori di resistenza forniti dall'Eurocodice sono stati incrementati di un fattore $f(R)=1.15$ (Eq. 2). Nel caso di provini forati (serie AL 1 e AL 3) il valore risultante è stato quindi abbattuto di un fattore 2.4 per tener conto dell'effetto di concentrazione delle tensioni (valore suggerito dall'Eurocodice stesso per la tipologia di intaglio considerata, ovvero foro circolare centrato di diametro $20 \mathrm{~mm}$ su provino di larghezza $60 \mathrm{~mm}$ ). Per quanto riguarda la UNI 8634 sono stati calcolati i valori di resistenza per R $=0.1$, tenendo conto delle diverse tipologie di lega analizzate. Nel caso di provini forati tale valore è stato abbattuto dello stesso coefficiente 2.4 suggerito dall'Eurocodice. Le due normative forniscono valori di resistenza molto simili che risultano in netto vantaggio di sicurezza nel caso della lega 6060 (Fig. 5), viceversa a sfavore di sicurezza per la lega 7012 (Fig. 6, 7).

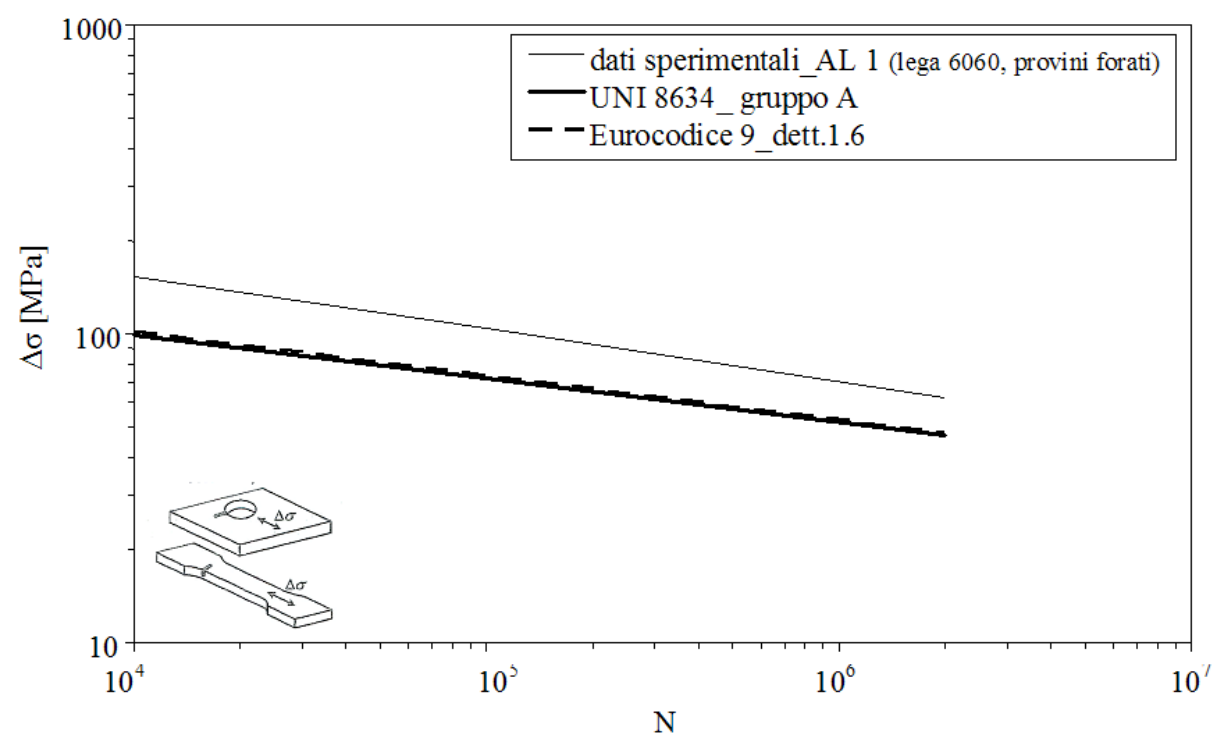

Figura 5: Confronto tra curve di resistenza a fatica per materiale base in lega 6060 (provini forati). 


\begin{tabular}{|c|c|c|c|c|c|}
\hline Serie & Tipologia di giunto & Fonte dato & $\begin{array}{l}\text { Riferimento- } \\
\text { Categoria dettaglio }\end{array}$ & $\begin{array}{l}\Delta \sigma[\mathrm{MPa}] \\
\mathrm{N}=2 \cdot 10^{6}, \\
\text { P.S. }=97.7 \%\end{array}$ & $\mathbf{k}$ \\
\hline \multirow[t]{3}{*}{ AL 1} & Materiale base & Sperimentale & Bellemo [25] & 62.4 & 5.9 \\
\hline & Lega P-Al-Mg-Si (6060) & UNI 8634 & Gruppo A & 47 & 7.1 \\
\hline & Provini forati & Eurocodice 9 & Dett. 1.6 & 47.9 & 7 \\
\hline \multirow[t]{3}{*}{ AL 2} & Materiale base & Sperimentale & Bellemo [25] & 45.1 & 3.7 \\
\hline & Lega Zergal 4 (7012) & UNI 8634 & Gruppo A & 110.7 & 7.1 \\
\hline & Provini lisci & Eurocodice 9 & Dett. 1.6 & 115 & 7 \\
\hline \multirow[t]{3}{*}{ AL 3} & Materiale base & Sperimentale & Bellemo [25] & 19.5 & 2.8 \\
\hline & Lega Zergal 4 (7012) & UNI 8634 & Gruppo A & 46.1 & 7.1 \\
\hline & Provini forati & Eurocodice 9 & Dett. 1.6 & 47.9 & 7 \\
\hline \multirow[t]{3}{*}{ AL 4} & Giunto testa a testa & Sperimentale & Van Straalen et al. [26] & 46.5 & 4.7 \\
\hline & & UNI 8634 & Gruppo D & 41.5 & 4.3 \\
\hline & & Eurocodice 9 & Dett. 7.3.1 & 40 & 4.3 \\
\hline \multirow{4}{*}{$\begin{array}{l}\text { AL } 5 \\
\text { AL } 6\end{array}$} & Lamiera con irrigidimento & Sperimentale & Meneghetti [27] $(12 / 10)$ & 47.4 & 4.2 \\
\hline & trasversale (giunto a $\mathrm{T}$ ) & & Ribeiro [28] (12/12) & 35 & 3.8 \\
\hline & & UNI 8634 & Gruppo D & 41.5 & 4.3 \\
\hline & & Eurocodice 9 & Dett. 3.1 & 32 & 3.4 \\
\hline \multirow{7}{*}{$\begin{array}{l}\text { AL } 7 \\
\text { AL } 8 \\
\text { AL } 9 \\
\text { AL } 10 \\
\text { AL } 11\end{array}$} & Lamiera con irrigidimenti & Sperimentale & Maddox [29] (3/3) & 45.2 & 3.7 \\
\hline & trasversali simmetrici & & Maddox [29] (6/6) & 37.3 & 4.3 \\
\hline & (giunto a croce, & & Maddox [29] (12/12) & 34.7 & 3.8 \\
\hline & cordone non portante) & & Maddox [29] (24/6) & 43.1 & 3.8 \\
\hline & & & Maddox [29] (12/6) & 39.3 & 3.7 \\
\hline & & UNI 8634 & Gruppo E & 36 & 4.3 \\
\hline & & Eurocodice 9 & Dett. 3.1 & 32 & 3.4 \\
\hline \multirow{4}{*}{$\begin{array}{l}\text { AL } 12 \\
\text { AL } 13\end{array}$} & Giunto a croce, & Sperimentale & Ribeiro [28] & 20.3 & 4.6 \\
\hline & cordone portante & & Jacoby [30] & 28 & 4.4 \\
\hline & (cordone d'angolo o & UNI 8634 & Gruppo F & 29.5 & 4.3 \\
\hline & parziale penetrazione) & Eurocodice 9 & Dett. 9.1 & 28 & 3.4 \\
\hline \multirow[t]{3}{*}{$\mathrm{Al} 14$} & Attacchi longitudinali & Sperimentale & Van Straalen et al. [31] & 25.6 & 3.3 \\
\hline & & UNI 8634 & Gruppo G & 19.5 & 4.3 \\
\hline & & Eurocodice 9 & Dett. 3.8 & 23 & 3.4 \\
\hline \multirow[t]{3}{*}{ Al 15} & Attacchi longitudinali & Sperimentale & Voutaz et al. [32] & 23.5 & 3.6 \\
\hline & su travi & UNI 8634 & Gruppo G & 19.5 & 4.3 \\
\hline & & Eurocodice 9 & Dett. 13.2 & 18 & 3.4 \\
\hline
\end{tabular}

Tabella4: Serie sperimentali analizzate: corrispondenza con le normative e confronto dei valori di resistenza.

\section{Giunti testa a testa}

I dati sperimentali fanno riferimento a giunti testa a testa saldati su un solo lato con ripresa. Lo spessore delle lamiere saldate è pari a $12 \mathrm{~mm}$. Le prove a fatica sono state realizzate in trazione, le rotture sono avvenute a piede del cordone di saldatura.

Come si evince da Fig. 8 le normative forniscono valori di resistenza molto simili e in accordo, con lieve margine di sicurezza, con i dati sperimentali.

Lamiera con irrigidimento trasversale (giunto a $T$ )

I dati sperimentali fanno riferimento a serie avente uguale spessore del piatto principale (12 mm) e spessori differenti della lamiera trasversale. Le prove a fatica sono state realizzate in trazione e le rotture si sono verificate a piede del cordone di saldatura. 
Per questa tipologia di giunto la normativa italiana fornisce dei valori di resistenza superiori a quelli dell' Eurocodice, come prevedibile tenendo conto che la UNI 8634 si basa su dati ricavati da provini. Mentre l'Eurocodice risulta a vantaggio di sicurezza, la UNI 8634 non lo è se si fa riferimento alla serie AL 5. I diversi valori di resistenza delle due serie non risultano imputabili a diversi campi di tensione locale (tali serie analizzate in termini di tensioni locali [17] hanno evidenziato uno stesso effetto di concentrazione delle tensioni dovuto alla geometria complessiva delle parti collegate e del cordone di saldatura, ovvero uno stesso valore del parametro $\mathrm{k}_{1}$ in Eq. 1) ma viceversa sembrano dovuti alla dispersione statistica dei risultati. Difatti i valori di $\Delta \sigma_{\mathrm{N}}=2 \cdot 10^{6}$, P.S. $=50 \%$ risultano rispettivamente di $57 \mathrm{MPa}$ per la serie AL 5 e $62 \mathrm{MPa}$ per la serie AL 6, tuttavia, mentre per la serie AL 5 il parametro di dispersione $\mathrm{T} \sigma=\Delta \sigma_{2.3 \%} / \Delta \sigma_{97.7 \%}$ risulta pari a 1.4 , per la serie AL 6 tale parametro risulta 3.1

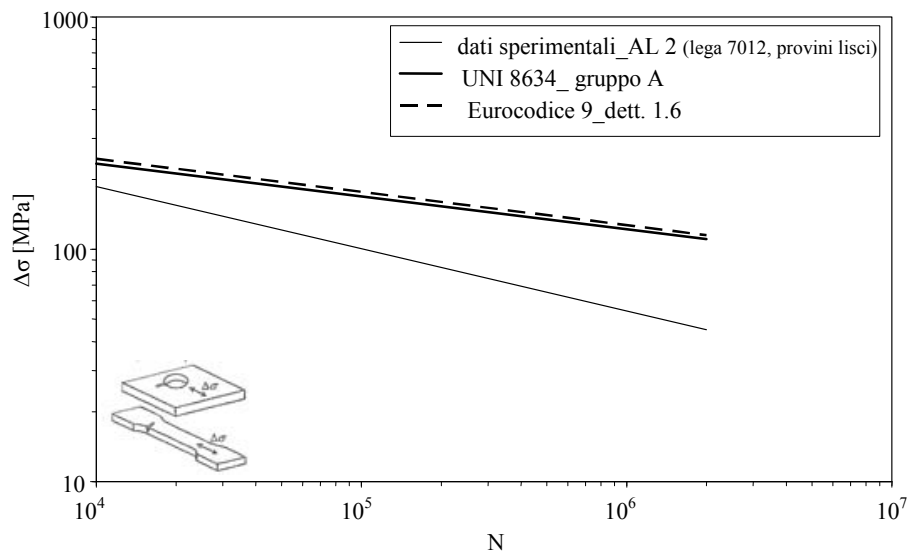

Figura 6: Confronto tra curva di resistenza a fatica per materiale base in lega 7012 (provini lisci).

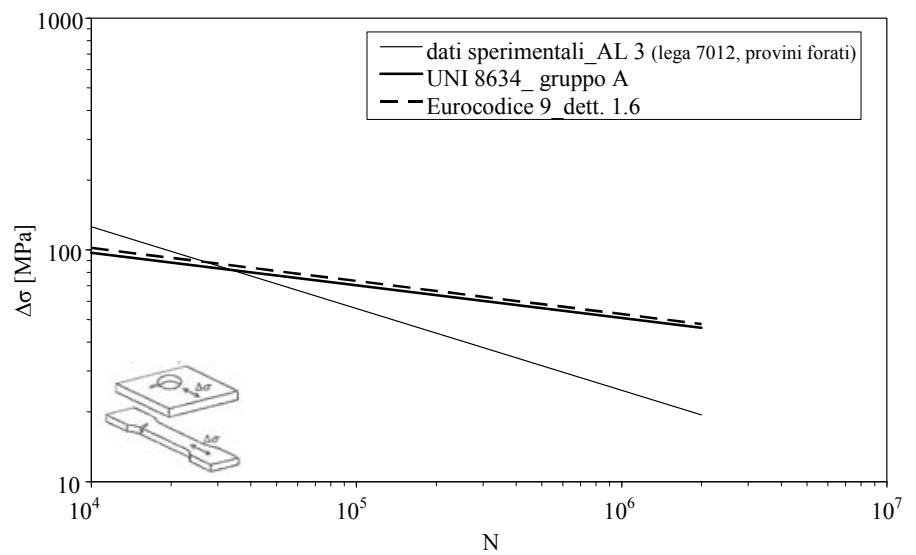

Figura 7: Confronto tra curva di resistenza a fatica per materiale base in lega 7012 (provini forati).

\section{Lamiera con irrigidimenti trasversali simmetrici (giunto a croce, cordone non portante)}

I dati sperimentali fanno riferimento a serie aventi diverse dimensioni delle parti saldate. Le prove a fatica sono state realizzate in trazione e tutte le rotture sono avvenute a piede del cordone di saldatura.

Dal grafico di Fig. 10 che riporta le curve di resistenza a fatica per giunti di uguale geometria (stessi rapporti L/t) ma dimensioni assolute diverse appare chiaramente come il non tener conto dell' effetto scala da parte sia dell'UNI 8634 che dell'Eurocodice 9 (che lo prevede solo per lunghezze della lamiera trasversale maggiori di 20 mm e con un esponente pari in media a circa 0.17 anziché 0.326 [22]), sebbene in parziale vantaggio di sicurezza, non permetta di apprezzare la variazione di resistenza associata a dimensioni assolute diverse. In Fig. 11 e 12 si illustrano invece le variazioni di resistenza associate a variazioni geometriche, ovvero variazioni dello spessore del piatto principale a parità di dimensioni della lamiera trasversale o viceversa. Tali variazioni, che vengono trascurate dalle normative, verrebbero invece messe in luce da un'analisi dei diversi campi di tensione locale [17] dovuti alle diversi geometrie di giunto pur all'interno di una medesima classe. Infatti per i giunti in Fig. 11 all'aumentare dello spessore del piatto principale il valore di $\mathrm{k}_{1}$ diminuisce. 
Analogamente, nel caso dei giunti in Fig. 12 l'aumento di spessore della lamiera trasversale comporta una maggiore gravosità del giunto indicata $\mathrm{da}$ un aumento del parametro $\mathrm{k}_{1}$ e quindi, a parità di spessore del piatto principale, in base all'Eq. 1, una diminuzione della resistenza a fatica. Si noti, con riferimento a quanto dimostrato da Meneghetti e Tovo [33] come l'utilizzo di un approccio di tipo hot spot per questa tipologia di giunti possa condurre a conclusioni errate.

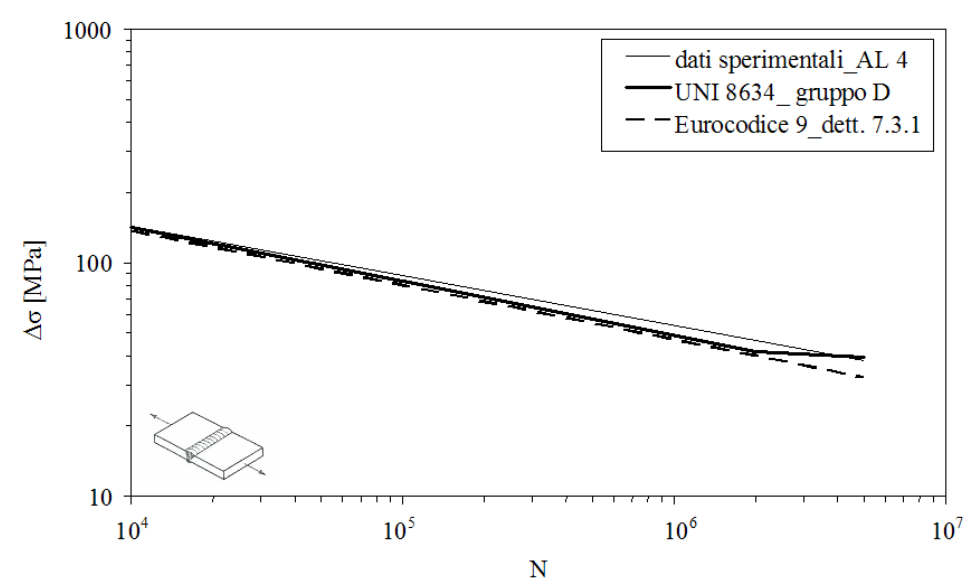

Figura 8: Confronto tra curva di resistenza a fatica per giunti testa a testa.

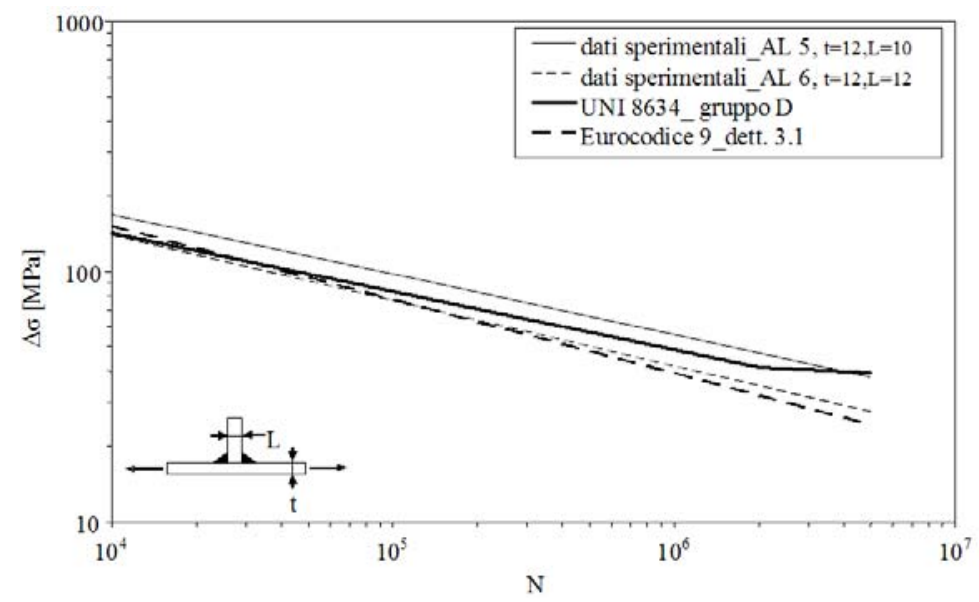

Figura 9: Confronto tra curva di resistenza a fatica per lamiera con irrigidimento trasversale (giunto a T).

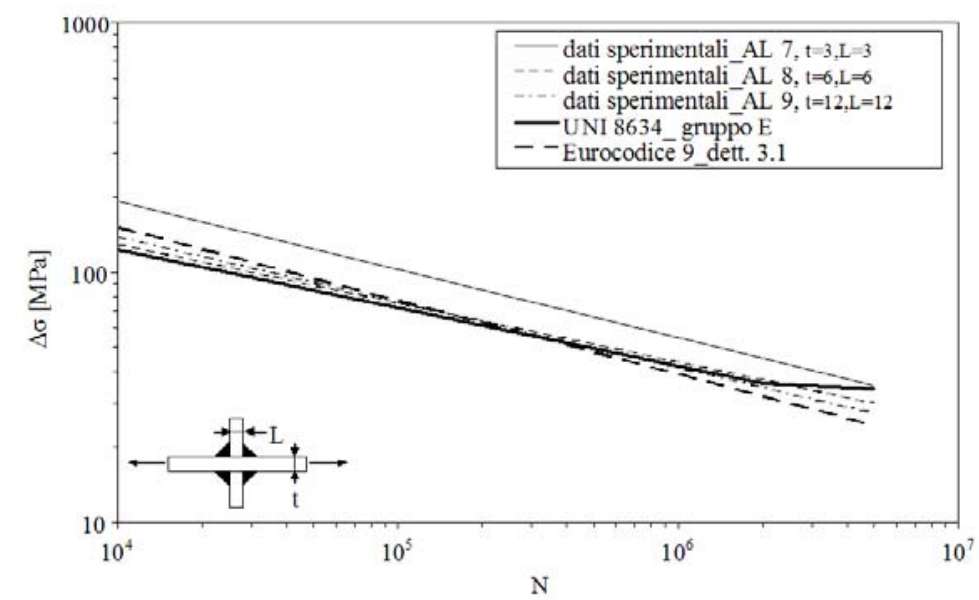

Figura 10: Confronto tra curva di resistenza a fatica per lamiere con irrigidimenti trasversali simmetrici (giunti a croce,cordone non portante). 


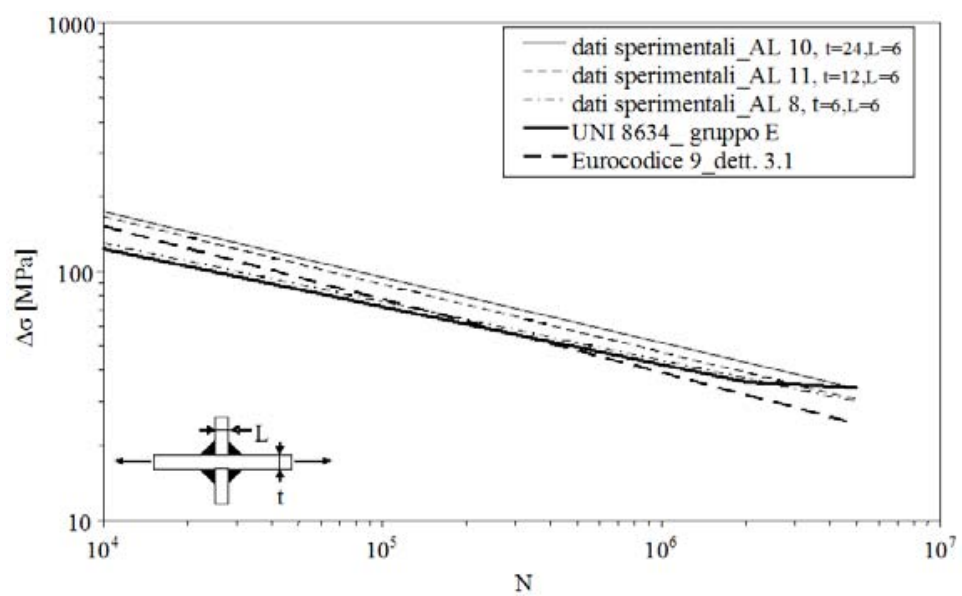

Figura 11: Confronto tra curva di resistenza a fatica per lamiere con irrigidimenti trasversali simmetrici (giunti a croce,cordone non portante).

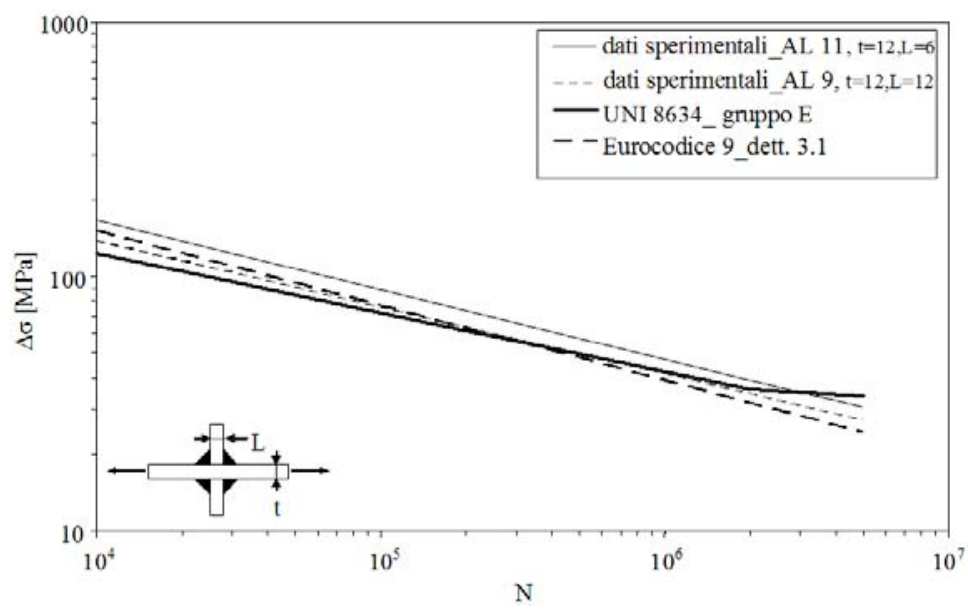

Figura 12: Confronto tra curva di resistenza a fatica per lamiere con irrigidimenti trasversali simmetrici (giunti a croce,cordone non portante).

Giunto a croce, cordone portante

I dati sperimentali fanno riferimento a due serie che differiscono unicamente per lo spessore del cordone di saldatura. Le prove a fatica sono state realizzate in trazione e le rotture sono avvenute a piede del cordone di saldatura.

La diversa criticità delle due serie, riscontrabile tramite un' analisi in tensioni locali [17] che evidenzia valori inferiori di $\mathrm{k}_{1}$ all'aumentare delle dimensioni del cordone di saldatura, non viene considerata dalle normative di progettazione che forniscono per altro delle curve a svantaggio di sicurezza. E' opportuno specificare tuttavia, che per i rapporti dimensioni del cordone e del piatto principale caricato presenti nelle due serie, l'Eurocodice prevede l'innesco di cricca alla radice del cordone di saldatura anziché al piede, con conseguente riduzione della classe di resistenza del giunto.

\section{Attacchi longitudinali}

I dati sperimentali fanno riferimento a due serie, la prima (AL 14) relativa ad attacchi longitudinali di lunghezza $120 \mathrm{~mm}$ saldati su piatto principale di lamiera di spessore $12 \mathrm{~mm}$ e testati in trazione, la seconda (AL 15) relativa ad attacchi longitudinali di lunghezza $200 \mathrm{~mm}$ saldati su una trave ad I (larghezza $101 \mathrm{~mm}$, altezza $216 \mathrm{~mm}$ ) avente spessore dell'ala $11 \mathrm{~mm}$ e testati in flessione. In entrambe le serie di prove le rotture si sono manifestate a piede del cordone di saldatura in direzione trasversale.

Entrambe le normative presentano delle curve di resistenza a vantaggio di sicurezza rispetto ai risultati sperimentali, con l'Eurocodice che fornisce dei valori di resistenza leggermente superiori rispetto alla UNI. La leggera diminuzione di 
resistenza della serie AL 15 rispetto alla serie AL 14 pare rendere ragione all'individuazione, nell'Eurocodice 9, di classi di resistenza differenti per attacchi longitudinali su lamiera o su travi.

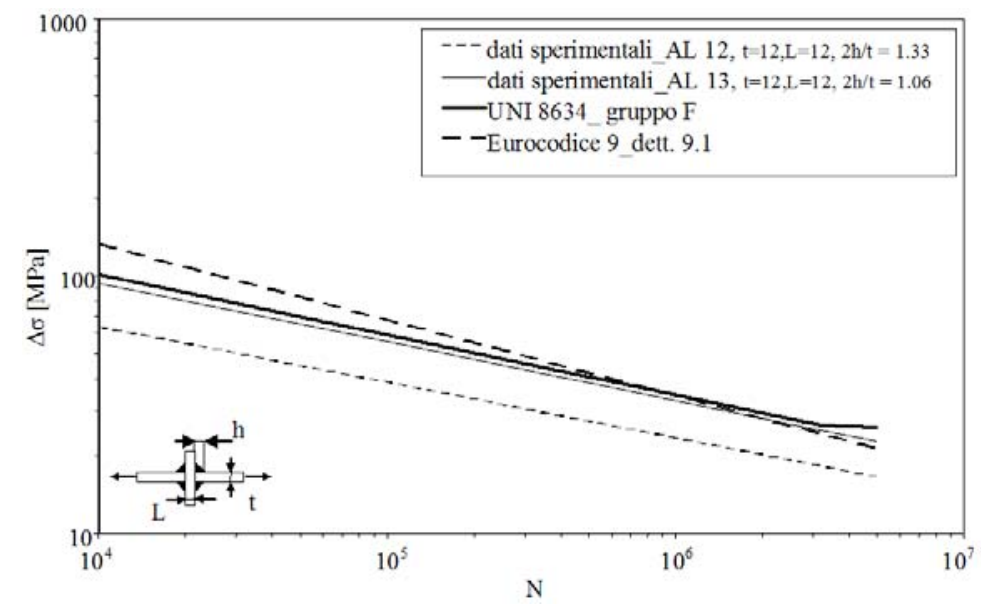

Figura 13: Confronto tra curva di resistenza a fatica per giunti a croce, cordone portante.

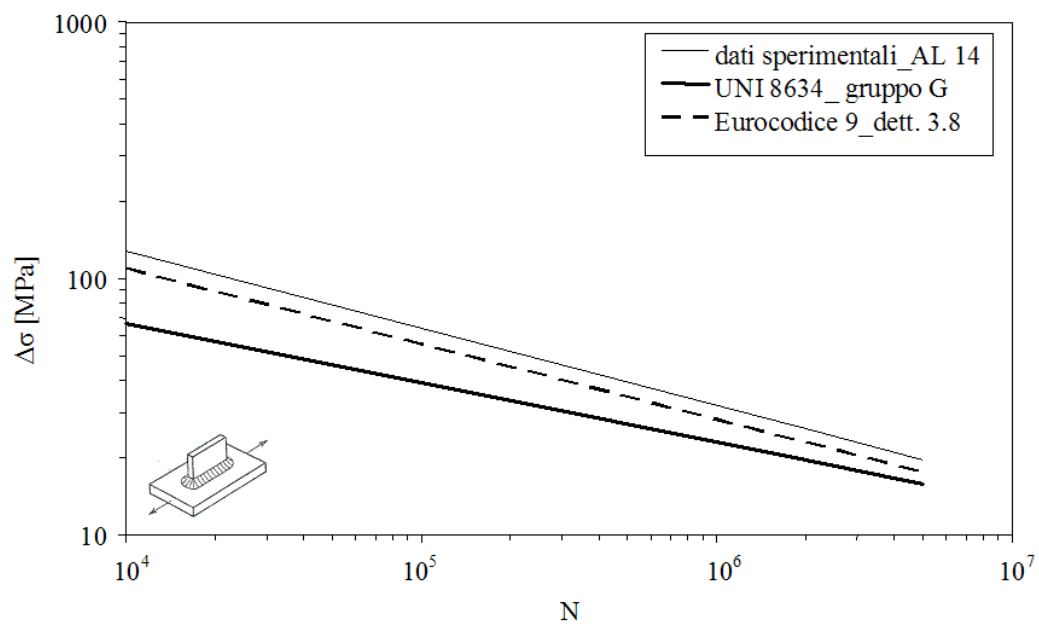

Figura 14: Confronto tra curva di resistenza a fatica per attacchi longitudinali.

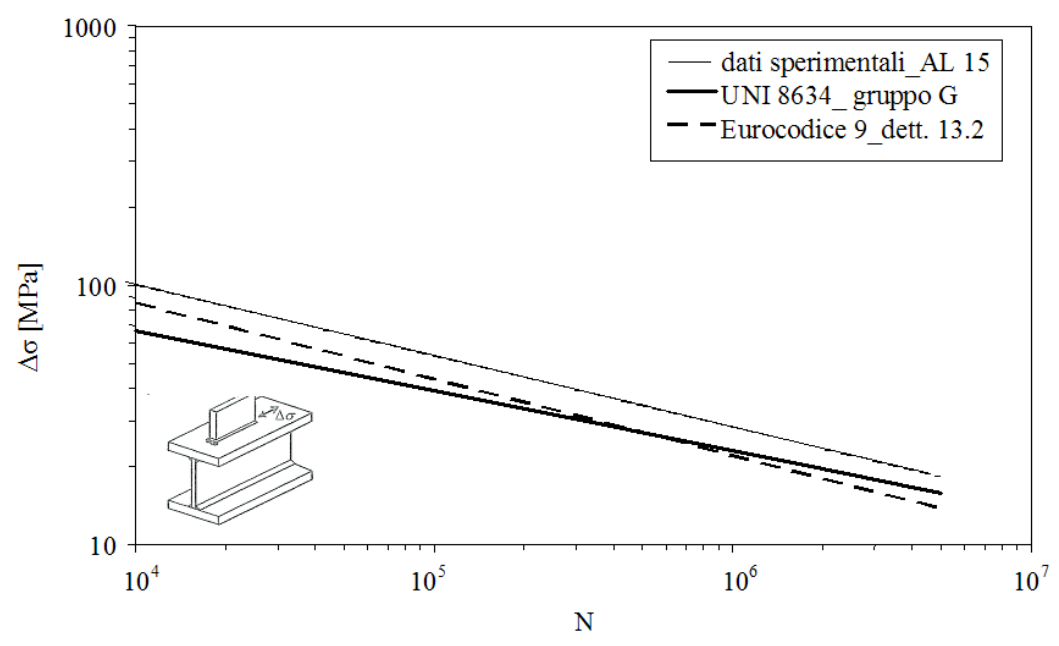

Figura 15: Confronto tra curva di resistenza a fatica per attacchi longitudinali su travi. 


\section{CONCLUSIONI}

$\mathrm{L}$

e curve di resistenza a fatica proposte dalla normativa italiana UNI 8634 e dall'Eurocodice 9 sono state confrontate con i valori di resistenza ricavati da dati sperimentali tratti da letteratura e riferiti a 15 serie di geometria diversa. Benché nella maggior parte dei casi in esame i valori forniti dalle normative o rispecchiano fedelmente i risultati sperimentali (giunti testa a testa), o si pongono a vantaggio di sicurezza (materiale base in lega 6060, giunti a croce non portante, attacchi longitudinali); in alcuni casi le curve di progettazione risultano pericolosamente a sfavore di sicurezza (materiale base in lega 7012, giunti a croce portante). In altri casi le due normative, seppure entrambe a vantaggio di sicurezza, forniscono valori di resistenza significativamente differenti tra loro (attacchi longitudinali) con conseguenti difficoltà nel passaggio da una normativa ad un'altra.

Il confronto con i dati sperimentali ha inoltre permesso di confermare un'ingiustificata sottovalutazione dell'effetto scala, e di mostrare come l'utilizzo dell'approccio in tensioni nominali non permetta di stimare variazioni di resistenza legate alla geometria complessiva sia del giunto che del cordone di saldatura e valutabili invece solo tramite un'analisi dei campi di tensione locali.

\section{BIBLIOGRAFIA}

[1] UNI 8634 Strutture in leghe di alluminio. Istruzioni per il calcolo e l'esecuzione (1985).

[2] E.Haibach, B. Atzori, LBF Rep. No. FB-116 (1974).

[3] E. Haibach, B. Atzori, Aluminium. (1975)

[4] B. Atzori, V. Dattoma, Proc. IIW Annual Assembly, IIW Doc. XIII-1088/3, Porto, Portugal (1981).

[5] CNR UNI 10011 Costruzioni in acciaio. Istruzioni per il calcolo, l'esecuzione, la manutenzione (1980).

[6] CNR UNI 10011 Costruzioni in acciaio. Istruzioni per il calcolo, l'esecuzione, la manutenzione (1986).

[7] UNI ENV 1999-2 Eurocodice 9. Progettazione delle strutture di alluminio. Parte 2: Strutture sottoposte a fatica (2002).

[8] UNI EN 1999-1-3 Eurocodice 9. Progettazione delle strutture di alluminio. Parte 1-3: Strutture sottoposte a fatica (2007).

[9] UNI EN 1993-19 Eurocodice 3. Progettazione delle strutture di acciaio. Parte 1-9: Fatica (2005).

[10] B. Atzori, B. Rossi, K. Heuler, Atti del XXVI Convegno Nazionale AIAS, Napoli (2007).

[11] B. Atzori, B. Rossi, Rivista Italiana della Saldatura 1 (2008) 93.

[12] B. Atzori, E. Haibach, Atti del XVII Convegno Nazionale AIAS, Cagliari (1979).

[13] B. Atzori, G. Blasi, C. Pappalettere, Exp. Mech., 25(2) (1985)129.

[14] P. Lazzarin, R. Tovo, Fatigue Fract. Engng Mater. Struct., 21 (1998) 1089.

[15] B. Atzori, P. Lazzarin, R. Tovo, Fatigue Fract. Engng Mater. Struct. 22 (5) (1999)369.

[16] B. Atzori, P. Lazzarin, R. Tovo, J. Strain Anal. Eng. 34 (1999) 437.

[17] P. Lazzarin, P. Livieri, Int. J. Fatigue, 23 (2001) 225.

[18] B. Atzori, G. Meneghetti, Int. J. Fatigue, 23 (8) (2001) 713.

[19] B. Atzori, G. Meneghetti, L. Susmel, Int. J. Fatigue, 24 (2002) 591.

[20] P. Livieri, P. Lazzarin, Int. J. Fracture, 133 (2005) 247.

[21] B. Atzori, G. Meneghetti, Proceedings of the International Conference of New Trends in Fatigue and Fracture, Metz (2002).

[22] B. Atzori, G. Meneghetti, M. Ricotta, Atti del XXXVI Convegno Nazionale AIAS, Napoli (2007).

[23] B. Atzori, P. Lazzarin, G. Meneghetti, M. Ricotta, Int. J. Fatigue, 31 (2009) 59.

[24] B. Atzori, Rivista Italiana della Saldatura, LII (1) (2000).

[25] S. Bellemo I materiali metallici nelle strutture soggette a fatica: confronto tra le leghe leggere e gli acciai. Tesi di laurea, Dipartimento di Ingegneria Meccanica, Università di Padova (1988).

[26] IJ. J. Van Straalen, F. Soetens, O. D. Dijkstra, TNO building and Construction Research-Report 94-CON-R1563 (1994).

[27] G. Meneghetti Metodologie di analisi e progettazione di giunti saldati. Tesi di dottorato, Università di Padova (1998).

[28] AS. Ribeiro, JP. Gonçalves, F. Oliveira, PT. Castro, AA. Fernandes, Proceedings of the Sixth International Conference on Aluminium Weldments, Cleveland, Ohio, (1995) 65.

[29] J. Maddox, Proceedings of the Sixth international Conference on Aluminium Weldments, Cleveland Ohio, (1995) 77. 
[30] G. Jacoby Über das verhalten von schweissverbindungen aus aluminiumlegierungen bei schwingbeanspruchung. Dissertation, Technische Hochschule, Hannover (1961).

[31] IJ. J. Van Straalen, F. Soetens, O. D. Dijkstra, TNO building and Construction Research-Report 94-CON-R1566 (1994).

[32] B. Voutaz, I.F. Smith, M.A. Hirt, Proceedings of the Third International Conference on Steel and Aluminium Structures, Istanbul, Turkey (1995).

[33] G. Meneghetti, R. Tovo, Proceedings INALCO Int. Conference, Cambridge, UK (1998). 